\title{
Comparison of digital versus fiberoptic cholangioscopy in patients requiring evaluation of bile duct disease or treatment of biliary stones
}

\author{
Ioannis D. Dimas ${ }^{a}$, Emmanouil Vardas a , Vasilios Papastergioua ${ }^{a}$ Maria Fragakia, Magdalini Velegrakia, \\ Afroditi Mpitoulia, Evangelos Voudoukisa , Angeliki Theodoropoulou ${ }^{a}$, Elpida Giannikaki ${ }^{b}$, \\ Gregorios Chlouverakisc, Gregorios A. Paspatis ${ }^{\mathrm{a}}$
}

Venizelion General Hospital, Heraklion, Crete; University of Crete, Heraklion, Crete, Greece

Abstract

\begin{abstract}
Background Recently, the introduction of the novel digital SpyGlass ${ }^{\mathrm{mw}}$ DS Direct Visualization system (Boston Scientific Corp., Natick, MA, USA) has signaled the transition into the era of digital singleoperator cholangioscopy (D-SOC). We sought to compare the clinical utility between fiberoptic single-operator cholangioscopy (F-SOC) and D-SOC in a tertiary-care referral center in Greece.

Methods This was a retrospective analysis of a prospective database of single-operator cholangioscopy (SOC) procedures performed over an 8-year period (2009-2017) at a single tertiary-care referral center. The study population consisted of consecutive adults referred for cholangioscopy for a variety of clinical indications, including biliary strictures, difficult biliary stones and migrated or occluded pancreatic or biliary stents.
\end{abstract}

Results A total of 2763 endoscopic retrograde cholangiopancreatography procedures were performed during the study period. Overall, SOC was performed in $68(2.46 \%)$ procedures (F-SOC $=39, \mathrm{D}-\mathrm{SOC}=29)$, showing a significant increase in the utilization of cholangioscopy during the D-SOC (29/599; 4.84\%) compared with the F-SOC (39/2124; $1.83 \%)$ period ( $\mathrm{P}=0.0001)$. The overall technical success of diagnostic SOC was $69.1 \%$ (38/55), being marginally higher for D-SOC (83.3\%) than for F-SOC (58.1\%), although not reaching statistical significance $(\mathrm{P}=0.07)$.

Conclusions D-SOC was utilized more frequently in our tertiary-care non-academic referral center, demonstrating a favorable safety profile and a trend towards a marginally higher technical success rate for the diagnosis of biliary strictures compared with F-SOC.

Keywords Endoscopic retrograde cholangiopancreatography, single-operator cholangioscopy, fiberoptic Spyglass, digital spyglass

Ann Gastroenterol 2019; 32 (2): 1-6

\section{Introduction}

Endoscopic retrograde cholangiopancreatography (ERCP) has been widely endorsed as the modality of choice for the

Departments of ${ }^{\mathrm{a}}$ Gastroenterology and ${ }^{\mathrm{b} P a t h o l o g y, ~ V e n i z e l i o n ~ G e n e r a l ~}$ Hospital, Heraklion, Crete, and 'Department of Social Medicine, University of Crete, Heraklion, Greece

Conflict of Interest: None

Correspondence to: Gregorios A. Paspatis, Venizelion General Hospital, Knossou Ave., 71409 Heraklion, Crete, Greece, e-mail: gpaspatis@gmail.com

Received 29 October 2018; accepted 9 January 2019; published online 25 January 2019

DOI: https://doi.org/10.20524/aog.2019.0358 diagnosis and treatment of biliary and pancreatic diseases, although it is not free of limitations. These include the suboptimal diagnostic performance of two-dimensional fluoroscopy; even therapeutic maneuvers, such as difficult stone removal, can be challenging to perform under fluoroscopic guidance only $[1,2]$.

Cholangioscopy was introduced as early as the 1970s [3,4], but the technology at that time presented insuperable impediments. In 2007, the emergence of the fiberoptic single-operator cholangioscopy (F-SOC) system (SpyGlass $^{\text {Tn }}$ Direct Visualization System, Boston Scientific Corp, Natick, MA, USA) was a major step forward that allowed cholangioscopic procedures to be performed by a single endoscopist. This system has also led to improved diagnostic sensitivity, offering direct visualization of the biliary and pancreatic ducts and the ability to obtain targeted biopsies $[5,6]$. Furthermore, the management of difficult stones has been greatly facilitated by the potential for interventional 
procedures, including electrohydraulic lithotripsy (EHL) or laser lithotripsy, or guidewire advancement in patients with biliary stricture [7]. However, poor stability, the lack of suction, a relatively small accessory channel and poor visibility were inherent limitations of the F-SOC system [6,8-11].

Recently, introduction of the novel digital SpyGlass ${ }^{\mathrm{mi}}$ DS Direct Visualization system (Boston Scientific Corp., Natick, MA, USA) has signaled the transition into the era of digital single-operator cholangioscopy (D-SOC). The advantages of this new system include: i) easy insertion into the biliary tract due to its tapered tip; ii) favorable visualization due to a high-resolution, integrated $120^{\circ}$ digital field of view; and iii) newly added injection and suction functions, carried out through a 2-port adaptor [12,13]. Moreover, a wide range of interventional procedures are made feasible through its accessory channel, including advanced methods for the management of difficult stones, tumor ablation, and control of bleeding $[14,15]$. In line with these advancements, Osanai et al, using a similar device (CHF-B260 and CHF-BP260 cholangioscopes; Olympus Medical Systems, Tokyo, Japan), recently determined that peroral video cholangioscopy may distinguish benign from malignant indeterminate strictures in $92.1 \%$ of patients, showing superior diagnostic accuracy compared with biopsy alone (diagnostic yield: 85.7\%) [16].

In the present study, we sought to compare the clinical utility between F-SOC and D-SOC in a tertiary-care referral center in Greece. To the best of our knowledge, only a single retrospective study, conducted in an academic setting, has directly compared the 2 modalities so far [17].

\section{Patients and methods}

\section{Patients}

This was a retrospective analysis of a prospective database of single-operator cholangioscopy (SOC) procedures performed over an 8-year period (May 2009 to March 2017) at a single tertiary-care referral center (Venizelion General Hospital, Heraklion, Crete). The study population consisted of consecutive adults referred for SOC for a variety of clinical indications, including biliary strictures, difficult biliary stones and migrated or occluded pancreatic or biliary stents. All patients had previously undergone noninvasive imaging techniques (e.g., computed tomography or endoscopic ultrasound), suspicious or suggestive of biliary disorders, but they could not be diagnosed or treated by conventional ERCP (i.e., ERCP with sphincterotomy or sphincteroplasty or both, in conjunction with balloon extraction and mechanical lithotripsy) performed in our hospital or another institution. Pregnancy, coagulation disorders, ineligibility for ERCP on clinical grounds, and inability to provide informed consent were exclusion criteria. Data recorded included demographics, procedure indication, intent of the procedure (diagnostic vs. therapeutic; patients with both diagnostic and therapeutic indications were placed in the therapeutic subgroup), therapeutic intervention, rate of complications, performance of SOC-directed biopsies and pathological findings. Institutional review board approval was obtained.

\section{SOC procedures}

All procedures were performed under monitored anesthesia care by 2 experienced endoscopists (EV, GAP) using standard technique $[9,18,19]$. Briefly, SOC is performed as adjunct to ERCP for the diagnosis and treatment of pancreatobiliary disorders. Sphincterotomy is usually carried out to better access the biliary tree, a guidewire is inserted under fluoroscopy, and Spyglass is passed over it into the bile or pancreatic duct. The guidewire is then removed to permit direct visualization of the duct through the repeated advancing and withdrawing of Spyglass. Between May 2009 and August 2015, only F-SOC was available, whereas between September 2015 and March 2017 cholangioscopy procedures were exclusively performed using D-SOC. All patients received intraprocedural antibiotics, continued for another 7 days. Biopsies were obtained using the forceps biopsy (SpyBite Boston Scientific, Natick, MA, USA), with approximately 3-8 biopsies per case. A single experienced pathologist (EG) performed all histopathological analyses, including evaluation of the adequacy of the obtained biopsy specimens.

The grade of technical difficulty was assessed using the Schutz technical difficulty scale: Grade 1, cannulation of the duct and stent removal or exchange; Grade 2, treatment of extrahepatic strictures, biliary leaks and stone extraction $<10 \mathrm{~mm}$; Grade 3, advanced procedures, such as extraction of larger stones, removal of migrated bile duct stents, treatment of benign or malignant strictures of the hilum and above, including intraductal biopsy under direct visualization; and Grade 4, intraductal therapy (e.g. EHL etc.), ERCP after Whipple procedure or Roux-en-Y surgery and removal of migrated pancreatic duct stents [20].

Cholangioscopic findings were judged to be malignant (dilated irregular tortuous vessels coursing through the epithelium, easy bleeding and irregular papillogranular or nodular surface protruding into the lumen of the bile duct) or benign (fine network of thin vessels, flat surface, homogeneous papillogranular surface without masses or white surface with convergence of folds) according to previously published criteria [12,21-23]. A final diagnosis of malignancy was based on histopathological diagnosis, including evaluation of surgical specimens, or cancer confirmation during a 6-month clinical follow up.

\section{Adverse events}

Post-ERCP pancreatitis was defined as a 3-fold elevation in serum amylase with concurrent abdominal pain after the procedure [24]. Cholangitis was defined according to the diagnostic criteria and severity assessment of acute cholangitis in the revised Tokyo Guidelines [25]. All others causes of fever/ infection were excluded in all cases.

\section{Outcome measures}

The present study primarily aimed to assess the comparative clinical utility of D-SOC versus F-SOC for diagnostic and therapeutic 
procedures. For this purpose, the technical success rate was compared between the D-SOC and F-SOC cohorts, defined as successful visualization of the target lesions in the biliary tract and carrying out forceps biopsy during diagnostic procedures, if needed [22].

For therapeutic procedures, technical success was defined as carrying out successful treatment, such as guidewire insertion into the area of interest, EHL, or migrated stent removal [22]. Secondarily, we evaluated: i) the diagnostic accuracy (proportion of correctly classified cases) of visual cholangioscopic appearances compared with a final diagnosis of malignancy as the reference standard; and ii) the rates of adverse events. In addition, we compared the utilization of SOC during ERCP procedures performed in the F-SOC (May 2009 to August 2015) and D-SOC (September 2015 to March 2017) periods.

\section{Statistical analysis}

Categorical variables were tested using corrected chi-square or 2-sided Fisher's exact tests for univariate comparisons, as appropriate. For continuous variables, summary statistics were calculated as mean \pm standard deviation and comparisons were analyzed using Student's $t$-test. A P-value $<0.05$ was considered statistically significant. All analyses were performed using SPSS version 24.0 (Chicago, IL, USA) statistical software.

\section{Results}

\section{Patients and utilization of SOC}

A total of 2763 ERCP procedures were performed during the study period. Overall, SOC was undertaken in $68(2.46 \%)$ procedures (F-SOC $=39, \mathrm{D}-\mathrm{SOC}=29$ ), showing a significant increase in the utilization of cholangioscopy in the D-SOC (29/599; 4.84\%) compared with the F-SOC (39/2124; 1.83\%) period $(\mathrm{P}=0.0001)$. Biliary strictures $(\mathrm{n}=51 ; 75 \%)$ and difficult biliary stones ( $\mathrm{n}=12$ patients; $17.6 \%$ ) were the most common indications for cholangioscopy, followed by less common indications: 2 patients had a stent migration ( 1 in the bile and 1 in the pancreatic duct), 2 patients had an obstructed bile duct metal stent, previously placed because of malignancy, and 1 patient presented with a bile duct foreign body (Kehr's T-tube remnant).

No significant differences were observed between the F-SOC and D-SOC cohorts as far as demographics and procedural characteristics were concerned, including the indications for SOC and the level of technical difficulty according to the Schutz scale (Table 1).

\section{Diagnostic SOC}

A total of 55 patients $(\mathrm{F}-\mathrm{SOC}=31, \mathrm{D}-\mathrm{SOC}=24)$ underwent diagnostic SOC during the study period; the baseline and procedural characteristics are summarized in Table 2 . The vast majority of procedures were performed for the evaluation of biliary strictures, located in the common hepatic duct and common bile duct. The F-SOC and D-SOC cohorts were comparable with respect to baseline characteristics, including demographics, indications for cholangioscopy and the level of difficulty of the procedures. The overall technical success of diagnostic SOC was $69.1 \%$ (38/55), being marginally higher for D-SOC $(83.3 \%)$ than for F-SOC, although not reaching statistical significance ( $83.3 \%$ vs. $58.1 \%, \mathrm{P}=0.07)$.

Among patients presenting with biliary strictures, $10 / 28$ and 9/19 had cholangioscopic features suggestive of malignancy in the F-SOC and D-SOC cohorts, respectively. Overall, 10 patients in the F-SOC cohort and 12 patients in the D-SOC cohort underwent forceps biopsies. On pathologic evaluation, the histological sample quality was considered adequate in $8(80 \%)$ biopsies obtained with F-SOC and in 11 (91.7\%) biopsies obtained with $\mathrm{D}-\mathrm{SOC}(\mathrm{P}=0.57)$. A total of 6 patients who underwent cholangioscopy for biliary strictures had a final diagnosis of biliary stones ( 4 in the F-SOC and 2 in the D-SOC cohort). Overall (by terms of histology or progression of the disease), malignancy was diagnosed in a total of 20 patients (11

Table 1 Baseline and procedural characteristics in the study population

\begin{tabular}{|c|c|c|c|}
\hline Characteristics & Total cohort $(n=68)$ & F-SOC cohort ${ }^{*}(n=39)$ & D-SOC cohort ${ }^{*}(n=29)$ \\
\hline Age, mean (SD) & $61.4( \pm 18.1)$ & $59.0( \pm 17.2)$ & $64.4( \pm 19.1)$ \\
\hline Male, n (\%) & $38(55.9 \%)$ & $19(48.7 \%)$ & $19(65.5 \%)$ \\
\hline $\begin{array}{l}\text { Indication of cholangioscopy, n (\%) } \\
\text { Biliary stricture } \\
\text { Biliary stones } \\
\text { Migrated stent } \\
\text { Stent occlusion } \\
\text { Foreign body }\end{array}$ & $\begin{array}{c}51(75 \%) \\
12(17.6 \%) \\
2(2.9 \%) \\
2(2.9 \%) \\
1(1.5 \%)\end{array}$ & $\begin{array}{c}30(76.9 \%) \\
4(10.3 \%) \\
2(5.1 \%) \\
2(5.1 \%) \\
1(2.6 \%)\end{array}$ & $\begin{array}{c}21(72.4 \%) \\
8(27.6 \%) \\
- \\
- \\
-\end{array}$ \\
\hline High Technical difficulty (Schutz 3 or 4 ), n (\%) & $66(97.1 \%)$ & $37(94.9 \%)$ & $29(100 \%)$ \\
\hline $\begin{array}{l}\text { Intent of cholangioscopy, n (\%) } \\
\text { Diagnostic } \\
\text { Therapeutic }\end{array}$ & $\begin{array}{l}55(80.9 \%) \\
13(19.1 \%)\end{array}$ & $\begin{array}{c}31(79.5 \%) \\
8(20.5 \%)\end{array}$ & $\begin{array}{c}24(82.8 \%) \\
5(17.2 \%)\end{array}$ \\
\hline
\end{tabular}

\footnotetext{
${ }^{*}$ P-values for all comparisons $>0.05$
}

F-SOC, fiberoptic single-operator cholangioscopy; D-SOC, digital single-operator cholangioscopy; SD, standard deviation 
Table 2 Baseline and procedural characteristics in patients who underwent diagnostic cholangioscopy

\begin{tabular}{|c|c|c|c|}
\hline Characteristics & F-SOC cohort $(\mathrm{N}=31)$ & D-SOC cohort $(\mathrm{N}=24)$ & P-value \\
\hline Age, mean (SD) & $61.2( \pm 15.5)$ & $64.2( \pm 19.1)$ & 0.51 \\
\hline Male, n (\%) & $17(54.8)$ & $15(62.5)$ & \\
\hline $\begin{array}{l}\text { Indication of cholangioscopy, } \mathrm{n}(\%) \\
\text { Biliary stricture } \\
\text { Biliary stones } \\
\text { Obstructed CBD metal stent }\end{array}$ & $\begin{aligned} 28 & (90.3) \\
2 & (6.5) \\
1 & (3.2)\end{aligned}$ & $\begin{array}{c}19(79.2) \\
5(20.8) \\
0(0)\end{array}$ & 0.20 \\
\hline High technical difficulty (Schutz 3 or 4), n (\%) & $30(96.8)$ & $24(100)$ & \\
\hline $\begin{array}{l}\text { Visual findings in biliary strictures, } \mathrm{n}(\%) \\
\text { Malignancy } \\
\text { Benign }\end{array}$ & $\begin{array}{l}10(35.7) \\
18(64.3)\end{array}$ & $\begin{array}{c}9(47.4) \\
10(52.6)\end{array}$ & 0.54 \\
\hline Patients with biliary strictures undergoing forceps biopsy, $\mathrm{n} / \mathrm{N}(\%)$ & $10 / 28(35.7)$ & $12 / 19(63.2)$ & 0.08 \\
\hline Patients with adequate biopsy specimens, n/N (\%) & $8 / 10(80)$ & $11 / 12(91.7)$ & 0.57 \\
\hline $\begin{array}{l}\text { Results of biopsy in patients with adequate specimens, } \mathrm{n} / \mathrm{N}(\%) \\
\text { Malignancy } \\
\text { Benign }\end{array}$ & $\begin{array}{l}1 / 8(12.5) \\
7 / 8(87.5)\end{array}$ & $\begin{array}{l}7 / 11(63.6) \\
4 / 11(36.4)\end{array}$ & 0.06 \\
\hline $\begin{array}{l}\text { Final diagnoses in biliary strictures, } \mathrm{n}(\%) \\
\text { Malignancy } \\
\text { Indeterminate stenosis } \\
\text { Stones } \\
\text { Primary sclerosing cholangitis } \\
\text { IgG4-related cholangitis } \\
\text { Other }\end{array}$ & $\begin{array}{c}11(39.3) \\
8(28.6) \\
4(14.3) \\
2(7.1) \\
1(3.6) \\
2(7.1)^{\star}\end{array}$ & $\begin{array}{c}9(47.3) \\
4(21.1) \\
2(10.5) \\
4(21.1) \\
0(0) \\
0(0)\end{array}$ & \\
\hline Technical success, $\mathrm{n}(\%)$ & $18(58.1)$ & $20(83.3)$ & 0.07 \\
\hline
\end{tabular}

and 9 in the F-SOC and D-SOC cohorts, respectively). The overall diagnostic accuracy of visual cholangioscopic appearances for differentiating malignant from benign biliary strictures was 95.0\% (19/20), with no significant difference between the F-SOC $(90.9 \% ; 10 / 11)$ and D-SOC $(100 \%$; 9/9) cohorts $(\mathrm{P}=0.99)$.

An analysis of malignancies in relation to biopsy data revealed that, when the fiberoptic system was used, in only 1 of 5 cases was the diagnosis confirmed histologically (20\%). In contrast, in all 7 patients of the D-SOC cohort who underwent biopsy the malignancy was confirmed histologically (100\%).

\section{Therapeutic SOC}

A total of 13 patients underwent therapeutic procedures (F-SOC $=8, \mathrm{D}-\mathrm{SOC}=5$ ) during the study period, most commonly for difficult bile duct stones. Two patients with migrated stents (1 biliary, 1 pancreatic), 1 patient who had an obstructed bile duct metal stent, 1 patient presenting with a bile duct foreign body, and 2 patients with stenosis needing guidewire passage underwent F-SOC (successful in 4/6 patients). Two patients with malignant stenosis successfully underwent D-SOC for guidewire advancement and radiofrequency ablation, respectively. Complete bile duct clearance was achieved in $1 / 2$ and $2 / 3$ patients undergoing therapeutic F-SOC and D-SOC, respectively. The overall technical success rate of therapeutic SOC was 5/8 (62.5\%) and 4/5 (80\%) for F-SOC and D-SOC, respectively ( $\mathrm{P}=0.99)$.

\section{Adverse events}

Adverse events are summarized in Table 3. Overall, there was no fatality directly associated with the procedures. Most diagnostic F-SOC procedures had no complications (25/31, $80.6 \%)$, there was no case of pancreatitis, but 6 presented cholangitis (19.4\%). Likewise, 22 of the 24 D-SOC diagnostic procedures presented no adverse events (91.6\%), 1 patient presented cholangitis (4.2\%) and there was 1 case of pancreatitis (4.2\%). There was no significant difference concerning the rate of adverse events between the diagnostic F-SOC and D-SOC groups $(\mathrm{P}=0.14)$. Likewise, no adverse events occurred in $6 / 8(75.0 \%)$ of therapeutic F-SOC and 4/5 (80\%) of therapeutic $\mathrm{D}$-SOC, respectively $(\mathrm{P}=0.99)$.

\section{Discussion}

Using a prospectively stored database, we determined that D-SOC was utilized twice as frequently compared with F-SOC. We hypothesize that better imaging quality, along with greater ease of setup and use, may have accounted for the increased utilization of D-SOC in our tertiary-care referral center. Likewise, improved technical aspects may justify the marginally higher, though not statistically significant, technical success rate of diagnostic D-SOC compared with F-SOC. However, 
Table 3 Adverse events associated with cholangioscopy

\begin{tabular}{lcccc}
\hline \multirow{2}{*}{ Adverse events } & \multicolumn{2}{c}{ Diagnostic cholangioscopy $(\mathrm{n}=55)$} & \multicolumn{2}{c}{ Therapeutic cholangioscopy $(\mathrm{n}=13)$} \\
\cline { 2 - 5 } & F-SOC $(\mathrm{n}=31)$ & D-SOC $(\mathrm{n}=24)$ & F-SOC $(\mathrm{n}=8)$ & 1 \\
\hline Cholangitis & 6 & 1 & 1 & 0 \\
Pancreatitis & 0 & 1 & 1 & 4 \\
\hline None & 25 & 22 & 6 & 1 \\
\hline
\end{tabular}

F-SOC, fiberoptic single-operator cholangioscopy; D-SOC, digital single-operator cholangioscopy

we could not detect any significant difference in terms of technical success rate when the 2 modalities were employed therapeutically. Ours is the second study to directly compare the 2 SOC modalities and the first to evaluate this comparison in a non-academic endoscopy setting.

In a previous retrospective study, Mizrahi et al also compared F-SOC with D-SOC [17]. They reported a significantly higher diagnostic yield of D-SOC in indeterminate strictures $(78 \%$ vs. $37 \%, \mathrm{P}=0.004)$, with a shorter procedure time and less radiation compared with F-SOC. Complementing the data of Mizrahi et al, our study addresses a trend towards increased technical success with diagnostic D-SOC, although a concrete difference in terms of diagnostic accuracy for distinguishing benign from malignant biliary strictures could not be detected in the current study. Clearly, this discrepancy may, at least partially, be explained by the lack of statistical power due to the relatively low number $(n=47)$ of patients with biliary strictures undergoing diagnostic SOC. Nevertheless, we would like to emphasize that our study was almost exclusively concerned with technically difficult cases, evaluated by a previously validated ERCP difficulty grading scale. Critically, no evaluation of technical difficulty was performed in the study by Mizrahi et al. Thus, comparisons between the 2 studies should be interpreted with caution, given the potential for significant heterogeneity, as well as the different endpoints used to comparatively assess the 2 SOC modalities.

On pathological evaluation, $80 \%$ of specimens obtained with F-SOC and $91.7 \%$ of those obtained with D-SOC were adequate for histology. These rates are roughly similar to those outlined by recent studies that evaluated each system separately $[6,26]$. Nevertheless, we demonstrated that for patients who had undergone biopsy with the fiberoptic system, in only 1 of 5 cases was the diagnosis confirmed histologically (20\%). In contrast, in all 7 patients in the D-SOC cohort who underwent biopsies the final diagnosis of malignancy was confirmed histologically (100\%). One limitation affecting targeted biopsies with the F-SOC system could be the limited field of view of the optical fiber and poor visualization.

Adverse events were comparable between the 2 systems. A retrospective study that compared ERCP with versus without cholangiopancreatoscopy ( $n=402$ vs. $n=3475$, respectively) showed increased morbidity if cholangiopancreatoscopy was performed [27]. In most cases, complications include cholangitis related to intraductal fluid irrigation; however, they are not clinically significant when treated with antibiotics $[23,27,28]$.

The present study is not free of limitations. First, it was performed at a single center, so results cannot be easily generalized. Second, the study was retrospective. Third, the number of patients included in each group was small. Fourth, the endoscopists were not blinded to the patients' clinical and laboratory data, this weakness being unavoidable. Finally, as per protocol, no scale was used for evaluation of the image quality of the 2 devices. On the other hand, evaluation of a prospectively stored database of ERCP procedures performed by 2 experienced endoscopists and the use of a validated technical difficulty scale may be considered as strengths.

In conclusion, D-SOC was utilized more frequently in our tertiary-care non-academic referral center, demonstrating a favorable safety profile and a trend towards a marginally higher technical success rate for the diagnosis of biliary strictures compared with F-SOC. The ease of use, along with the digital optics and improved suction and irrigation abilities of D-SOC, are expected to dramatically enhance the application of cholangioscopy in clinical practice.

\section{Summary Box}

\section{What is already known:}

- The introduction of cholangioscopy has overcome the inherent limitations of endoscopic retrograde cholangiopancreatography in the diagnosis and treatment of biliary and pancreatic diseases

- The emergence of fiberoptic single-operator cholangioscopy (F-SOC) improved diagnostic sensitivity and therapeutic potential

- The existing literature lacks adequate evidence concerning the superiority of the new digital singleoperator cholangioscopy (D-SOC), given the digital system's advantages compared with the fiberoptic

\section{What the new findings are:}

- Our tertiary-care non-academic referral center experience has demonstrated more frequent use of D-SOC

- Compared with F-SOC, D-SOC offers a favorable safety profile and a trend towards a marginally higher technical success rate in the diagnosis of biliary strictures 


\section{References}

1. Chathadi KV, Chandrasekhara V, Acosta RD, et al; ASGE Standards of Practice Committee. The role of ERCP in benign diseases of the biliary tract. Gastrointest Endosc 2015;81:795-803.

2. Anderson MA, Appalaneni V, Ben-Menachem T, et al; American Society for Gastrointestinal Endoscopy (ASGE) Standards of Practice Committee. The role of endoscopy in the evaluation and treatment of patients with biliary neoplasia. Gastrointest Endosc 2013;77:167-174.

3. Rösch W, Koch H. Peroral cholangioscopy in choledochoduodenostomy - patients using the pediatric fiberscope. Endoscopy 1978;10:195-198.

4. Urakami Y, Seifert E, Butke H. Peroral direct cholangioscopy (PDCS) using routine straight-view endoscope: first report. Endoscopy 1977;9:27-30.

5. Draganov PV, Chauhan S, Wagh MS, et al. Diagnostic accuracy of conventional and cholangioscopy-guided sampling of indeterminate biliary lesions at the time of ERCP: a prospective, long-term follow-up study. Gastrointest Endosc 2012;75:347-353.

6. Chen YK, Parsi MA, Binmoeller KF, et al. Single-operator cholangioscopy in patients requiring evaluation of bile duct disease or therapy of biliary stones (with videos). Gastrointest Endosc 2011;74:805-814.

7. Theodoropoulou A, Vardas E, Voudoukis E, et al. SpyGlass direct visualization system facilitated management of iatrogenic biliary stricture: a novel approach in difficult cannulation. Endoscopy 2012;44 Suppl 2 UCTN: E433-E434.

8. Kurihara T, Yasuda I, Isayama H, et al. Diagnostic and therapeutic single-operator cholangiopancreatoscopy in biliopancreatic diseases: prospective multicenter study in Japan. World $J$ Gastroenterol 2016;22:1891-1901.

9. Manta R, Frazzoni M, Conigliaro R, et al. SpyGlass single-operator peroral cholangioscopy in the evaluation of indeterminate biliary lesions: a single-center, prospective, cohort study. Surg Endosc 2013;27:1569-1572.

10. Navaneethan U, Hasan MK, Lourdusamy V, Njei B, Varadarajulu S, Hawes RH. Single-operator cholangioscopy and targeted biopsies in the diagnosis of indeterminate biliary strictures: a systematic review. Gastrointest Endosc 2015;82:608-614.e2.

11. Ramchandani M, Reddy DN, Gupta R, et al. Role of single-operator peroral cholangioscopy in the diagnosis of indeterminate biliary lesions: a single-center, prospective study. Gastrointest Endosc 2011;74:511-519.

12. Parsi MA, Jang S, Sanaka M, Stevens T, Vargo JJ. Diagnostic and therapeutic cholangiopancreatoscopy: performance of a new digital cholangioscope. Gastrointest Endosc 2014;79:936-942.

13. Parsi MA, Stevens T, Bhatt A, Jang S, Vargo JJ. Digital, catheter-based single-operator cholangiopancreatoscopes: can pancreatoscopy and cholangioscopy become routine procedures? Gastroenterology 2015;149:1689-1690.
14. Ogura T, Takagi W, Ueno S, Takeuchi T, Fukunishi S, Higuchi K. Endoscopic hemostasis for tumor bleeding using intraductal radiofrequency ablation. Endoscopy 2016;48:E328-E329.

15. Wong JC, Wong MY, Lam KL, Lau JY. Second-generation peroral cholangioscopy and holmium:YAG laser lithotripsy for rescue of impacted biliary stone extraction basket. Gastrointest Endosc 2016;83:837-838.

16. Osanai M, Itoi T, Igarashi $\mathrm{Y}$, et al. Peroral video cholangioscopy to evaluate indeterminate bile duct lesions and preoperative mucosal cancerous extension: a prospective multicenter study. Endoscopy 2013;45:635-642.

17. Mizrahi M, Khoury T, Wang Y, et al. "Apple far from the tree": comparative effectiveness of fiberoptic single-operator cholangiopancreatoscopy (FSOCP) and digital SOCP (DSOCP). HPB (Oxford) 2017;20:285-288.

18. Chathadi KV, Chen YK. New kid on the block: development of a partially disposable system for cholangioscopy. Gastrointest Endosc Clin N Am 2009;19:545-555.

19. Chen YK, Pleskow DK. SpyGlass single-operator peroral cholangiopancreatoscopy system for the diagnosis and therapy of bile-duct disorders: a clinical feasibility study (with video). Gastrointest Endosc 2007;65:832-841.

20. Schutz SM. Grading the degree of difficulty of ERCP procedures. Gastroenterol Hepatol (N Y) 2011;7:674-676.

21. Itoi T, Neuhaus H, Chen YK. Diagnostic value of image-enhanced video cholangiopancreatoscopy. Gastrointest Endosc Clin N Am 2009; 19:557-566.

22. Ogura T, Imanishi M, Kurisu Y, et al. Prospective evaluation of digital single-operator cholangioscope for diagnostic and therapeutic procedures (with videos). Dig Endosc 2017;29:782-789.

23. Tringali A, Lemmers A, Meves V, et al. Intraductal biliopancreatic imaging: European Society of Gastrointestinal Endoscopy (ESGE) technology review. Endoscopy 2015;47:739-753.

24. Cotton PB, Eisen GM, Aabakken L, et al. A lexicon for endoscopic adverse events: report of an ASGE workshop. Gastrointest Endosc 2010;71:446-454.

25. Kiriyama S, Takada T, Strasberg SM, et al; Tokyo Guidelines Revision Committee. New diagnostic criteria and severity assessment of acute cholangitis in revised Tokyo Guidelines. J Hepatobiliary Pancreat Sci 2012;19:548-556.

26. Navaneethan U, Hasan MK, Kommaraju K, et al. Digital, single-operator cholangiopancreatoscopy in the diagnosis and management of pancreatobiliary disorders: a multicenter clinical experience (with video). Gastrointest Endosc 2016;84:649-655.

27. Sethi A, Chen YK, Austin GL, et al. ERCP with cholangiopancreatoscopy may be associated with higher rates of complications than ERCP alone: a single-center experience. Gastrointest Endosc 2011;73:251-256.

28. Shah RJ. Innovations in intraductal endoscopy: cholangioscopy and pancreatoscopy. Gastrointest Endosc Clin N Am 2015;25:779-792. 